\title{
New Decentralized MRAC Algorithms for Large-scale Uncertain Dynamic Systems
}

\author{
A-Cheng $\mathrm{Wu}^{*}$ \\ Li-Chen $\mathrm{Fu}^{\dagger}$
Keywords: model reference adaptive control, averaging filter, interconnected dynamic systems, robustness

\begin{abstract}
In this paper, we present the first complete solution to the decentralized model reference adaptive control (MRAC) problem for general interconnected uncertain dynamic systems with arbitrarily large interconnection strengths. Fach local subsystem may be perturbed by significant unmodeled dynamics as well as contaminated by bounded output disturbances. Despite these, excellent properties of the closed-loop controlled systems can be proven, which are hardly achieved by conventional decentralized MRAC $[5,7]$. The tracking errors can be made as small as possible by simply decreasing the design time constant $\tau$. It should also be noted that the presented algorithms form the decentralized counterpart of our earlier work [12, 13, 14], where the variable structure design (VSD) for the MRAC of general uncertain plants is investigated. A simple example is also provided to explain the advantage of reducing the controller complexity when the proposed decentralized MRAC design is incorporated.
\end{abstract}

\section{Introduction}

The problem of decentralized adaptive control is a research subject of great theoretical and practical interests $[3,5,7],[9]$ [11]. Very recently, an attempt [10] has been made to deal with the design of decentralized adaptive controller for interconnected uncertain dynanic systems, where the uncertain dynamics are typically described by transfer functions perturbed by parametric, nonparametric uncertainties, and uncert ain interconnections. In contrast to the stochastic control approach, no assumptions are made concerning the statistics of the uncertainties considered. Alternatively, the approach is based on the given nominal plants which are subjected to parametric uncertainties, and the objective is to construct the controllers which ensure desirable performance in a deterministic sense (e.g. ultimate boundedness or uniform boundedness). In such a system, each local subsystem is controlled independently using an adaptive algorithm. Practical areas of application of this design philosophy include large-scale interconnected uncertain systems, for instance, power generation plants and process control systems [3.8].

It is known [9]-[11] that direct applications of morel reference adaptive controllers, $\leftrightarrow$ g. $[5,7]$, to the interconnected uncertain subsystems yield unsatisfactory system behavior arising from the poor knowledge of the system parameters, the unmodeled dynamics, and/or the unmodeled interconnections. A way to remedy this is to incorporate the VSD $[6]$ concept into the conventional decentralized design. A new approach to decentralized MRAC is recently developed [9] which achieves finitetime convergence of the tracking error despite the existence of

*Department of Electrical Engineering, NTU, Taipei, 107, TAIWAN, R.O.C.

${ }^{\dagger}$ Department of Electrical Engineering, Computer Science and Information Engineering, N'T'U, Taipei, 107, TAIWAN, R.O.C. interconnections among subsystems, which may be nonlinear and time-varying with any possible strengths. As a counterpart, we allow the exchange of the output information among subsystems to generate a normalizing signal so as to circumvent the effects of unmodeled dynamics and unmodeled interconnections [10]. To the best of our knowledge, it is the first work which ensures the finite-time perfect tracking property in the possible presence of unmodeled dynamics, unmodeled interconnections, and output disturbances. In the recent work [3], although the same interconnection pattern as that formulated in [9]-[11] is considered, the tolerable strength of the interconnection can only be $\mu$-small. Another related work [8] also proposed a decentralized adaptive scheme for systems of arbitrary relative degree without matching assumptions, but only for the purpose of stabilization.

In the present paper, based only on $1 / 0$ measurements, we develop novel decentralized MRAC algorithms for a class of interconnected uncertain dynamic systems in order to

(a) improve the transient behavior and the tracking performance by incorporating the VSD for each nominal subplant with any possible relative degree,

(b) counteract or reject the possible unmodeled dynamics, nonlinear time-varying interconnections, and/or output disturbances,

(c) reduce the computational difficulties due to the use of centralized algorithms, which may lead to computation or numerical instability,

We also point out that these proposed algoritlums form decentralized counterpart of our earlier results $[12,13,14]$. Note that the underlying framework can lead to more reliable control effects under normal operations as well as in some occasions with failure of the interconnections and/or the data transmitting network.

The layout of this paper is as follows: Section 2 motivates the problem of investigating the decentralized controller design through an illustrative example. Preliminaries and problem statements are given in section 3 . Practical redesign of the robust decentralized MRAC algorithm using averaging filter is established in section 4 . Simulation results for strongly connected t.wo-by-two plants are shown in section 5 . Section 6 concludes the paper by summarizing the main results and outlines our future research.

\section{Problem Motivation}

The MRAC for the continuous-time decentralized systems based only on $1 / O$ measurements has caused great research interests for a long time. In the case where each subsystem is of relativedegree one, some structural constraints are imposed [5] to make the stability of the overall system under the decentralized adaptive control be invariant to interconnections among subsystems. Incorporating the VS design concept, Wu et al. [9] proposed a completely decentralized MRAC scheme that hits the sliding mode of each subsystem, interconnected with arbitrarily large, 
possibly nonlinear and time-varying strengths, in fimite time. In [10], the decentralized MRAC for plants with additive, multiplicative unmodeled dynamics, and output disturbances are presenter. The finite-time perfect tracking property is still obtained the same as in [9] modulo some chattering around the ideal sliding mode. Under the assumptions that the high frequency gain of each of the isolated subsystem is known and the ummodeled intercomections are "small", Datta [3] shows that a clesign is possible in the case of decentralized MRAC with allowable exchange of output information arnong individual subsystems. However, the completely deceniralized MRAC: i.e, cannot allow the information exchanges among subsystems, for general plants with large interconnections is still an open question there. Before solving this, we will first give an numerical example to motivate the investigation of this decentralized controller design problem.

Consicier an example with two inputs and two outputs :

$$
\begin{aligned}
& y_{1}=G_{1}(s)\left(u_{1}\right)+H_{12}(s)\left(y_{2}\right) \\
& y_{2}=G_{2}(s)\left(u_{2}\right)+H_{21}(s)\left(y_{1}\right),
\end{aligned}
$$

where $u_{1}$ and $u_{2} \in R$ are the plant inputs, $y_{1}$ and $y_{2} \in R$ are the plant outputs, and $G_{1}(s)=\frac{1}{s-1}, G_{2}(s)=\frac{1}{s^{2}-1}, H_{12}(s)=$ $\frac{1}{(s+1)^{2}}, H_{21}(s)=\frac{1}{(s+1)^{3}}$ are plant transfer functions. For a better viewpoint, we define the compact vectors $u=\left[u_{1} u_{2}\right]^{T}$. $y=\left[y_{1} y_{2}\right]^{T}$, and the compact transfer function $G(s)$ as :

$$
\begin{aligned}
y & =G(s) u \\
& =\left[\begin{array}{ll}
\frac{G_{1}}{1-H_{12} H_{21}} & \frac{H_{12} G_{2}}{1-H_{1} H_{21}} \\
\frac{H_{21} G_{1}}{1-H_{12} H_{21}} & \frac{G_{2}}{1-H_{12} H_{21}}
\end{array}\right] u,
\end{aligned}
$$

which yields

$$
G(s)=\frac{1}{d(s)}\left[\begin{array}{cc}
(s+1)^{5} & (s+1)^{2} \\
10(s+1)^{2} & (s+1)^{4}
\end{array}\right]
$$

with $d(s)=(s-1)\left(s^{5}+5 s^{4}+10 s^{3}+10 s^{2}+5 s+9\right)$. Since the observability index of $G(s)$ is 6 , the total number of control parameters required by using the conventional scheme in [2] is $2 \times 6 \times 4=48$. But if we can directly apply the control scheme proposed in section 3.4 .2 to each subsystem (wherein one subsystem is with relative-degree two), the total number of control parameters is equal to $2 \times 1+2 \times 2=6$. This shows that the decentralized adaptive scheme is much less complex than the centralized adaptive scheme and is much more suitable for practical application. This explains the advantage and, hence, motivates the study of the decentralized controller design.

\section{Preliminaries and Problem Statements}

Consider a large-scale interconnected uncertain system containing $N$ SISO subsystems :

$$
\begin{aligned}
\Omega_{i}: y_{i}= & {\left[G_{o i}(s)\left(1+\mu_{1} \Delta_{m i}(s)\right)+\mu_{1} \Delta_{a i}(s)\right]\left(u_{i}\right)+d_{o i}(t) } \\
& +\mu_{2} \sum_{\substack{j=1 \\
i \neq j}}^{N} H_{i, j}(s)\left(F_{i j}\left(y_{j}, t\right)\right), \quad i \in \mathbf{N}
\end{aligned}
$$

where $\mathbf{N}=\{1, \cdots, N\}$, and for the i-th subsystem $\Omega_{i}, u_{i}(t)$. $y_{i}(t)$, and $\bar{d}_{o i}(t) \in R$ are plant input, plant output, and plant disturbance, respectively, $\Delta_{3 n i}(s)$ and $\Delta_{a i}(s)$ are the multiplicative and additive unmodeled dynamics, respectively, and $\mu_{1}$ and $\mu_{2}$ are positive constants. The function $F_{i j}\left(y_{j}, l\right): R \times R \rightarrow R$ stands for the interconnection input arising from the subsystem $\Omega_{j}$ to subsystem $\Omega_{i}$ satisfying

$$
\left|F_{i j}\left(y_{j}, t\right)\right| \leq \alpha_{i j}\left|y_{j}\right|, \quad j \in \mathbf{N}
$$

for some nonnegative numbers $\alpha_{i j}, j \neq i, i, j \in \mathbf{N}, H_{i j}(s)$ is the trausfer function from $F_{i j}\left(y_{j}, t\right)$ to $y_{i}$, and the nominal subplant $G_{o i}(s)=k_{p i} \frac{\hat{n}_{p i}(s)}{\hat{d}_{p i}(s)}$ is subjected to the following assumptions.

(S1) $G_{o i}(s)$ is of known relative degree $\nu_{i} \geq 2$ and $G_{o i}\left(s-q_{i}\right)$ is minimum plrase for some $q_{i}>0$, i.e., $\hat{n}_{p i}(s)$ and $\hat{d}_{p_{i}}(s)$ are both monic, coprime polynomials of degree $n_{i}-\nu_{i}$ and $n_{i}$, respectively, and $\hat{n}_{p i}\left(s-q_{i}\right)$ is Hurwilz.

(S2) The sign of $k_{p i}$ is known a priori and, without loss of gencrality, is assumed to be positive.

(S3) $\frac{\Delta_{m i}(s)}{\left(s+\alpha_{i}\right)^{\nu_{i}}}$ and $s \Delta_{a i}(s)$ are strictly proper and stable for some $\alpha_{i}>0$ and that $G_{i}(s)=G_{o i}(s)\left(1+\mu_{1} \Delta_{\text {ini }}(s)\right)+$ $\mu_{1} \Delta_{a i}(s)$ is minimum phase.

(S4) $d_{o i}(t)$ and $d_{o i}(t)$ are uniformly bounded.

(S5) $s H_{i j}\left(s-q_{i}\right)$ or $G_{o i}^{-1}\left(s-q_{i}\right) H_{i j}\left(s-q_{i}\right) /\left(s+\alpha_{i}\right)^{\nu_{i}}$ are stable and $s^{\nu_{i}} H_{i j}(s)$ is strictly proper.

Remarks 3.1 :

The dynamics of the interconnected systems $\Omega$ are unknown in the sense that the transfer function $G_{i}(s)$ and $H_{i j}(s)$ and the nonlinear time-varying functions $F_{i j}\left(y_{j}, t\right), j \neq i, i, j \in \mathbf{N}$ are not specified in advance. The plant model (3.1) shows that the interconnections to each subsystem are not necessary to appear in its control channel. As a consequence, $H_{i j}(s), j \neq i, i, j \in \mathbf{N}$ are in general different from $G_{i}(s)$, but when viewing from the state space model $[5,7]$ it is rational to assume that they should contain the same unstable poles as stated in (S5).

The tracking problem for the i-th subsystem (3.1) can be formulated as follows: design a control $u$; such that the local output $y_{i}$ tracked as closely as desired the reference output $y_{m i}$ of the local reference model :

$$
y_{m i}=M_{i}(s)\left(r_{i}\right)=k_{m i} \frac{\hat{n}_{m i}(s)}{\hat{d}_{m i}(s)}\left(r_{i}\right)
$$

In order to meet the control objective, we impose the following assumption on the reference model.

(S6) $M_{i}\left(s-q_{i}\right) L_{1 i}\left(s-q_{i}\right)$ is strictly positive real(SPR) [2] with $\hat{n}_{m i}(s)$ and $\hat{d}_{m i}(s)$ being monic Hurwitz polynomials of degree $n_{i}-\nu_{i}$ and $n_{i}$, respectively, $L_{1 i}(s)=$ $\left(s+a_{1 i}-q_{i}\right) \cdots\left(s+a_{\nu_{i}-1, i}-q_{i}\right)$ being a $\left(\nu_{i}-1\right)$-th order Hurwitz polynomial, $\operatorname{sgn}\left(k_{p}\right)=\operatorname{sgn}\left(k_{m}\right)$, and $r_{i}$ being the reference input.

For each subsystem, the model matching [1] between the isolated nominal transfer function $G_{o i}(s)$ and the reference transfer function $M_{i}(s)$ is given as :

$$
1-\bar{C}_{p i}^{*}(s)-\bar{D}_{f i}^{*}(s) G_{\imath \hat{\imath} i}(s)=\mathrm{c}_{o i}^{*} M_{i}^{-1}(s) G_{o i}(s)
$$

Following the same steps as shown in [10], we derive the error model for the intercomected subplants as:

$$
\begin{gathered}
e_{o i}=\frac{1}{c_{o i}^{*}}\left\{u_{i}-c_{o i}^{*} r_{i}-\bar{C}_{p i}^{*}(s)\left(u_{i}\right)-\bar{D}_{f i}^{*}(s)\left(y_{i}\right)+\left(1-\bar{C}_{p i}^{*}(s)\right)\right. \\
{\left[\mu_{1} \Delta_{m i}(s)\left(u_{i}\right)+\mu_{1} G_{m i}^{-1}(s) \Delta_{m i}(s)\left(u_{i}\right)+G_{o i}^{-1}(s)\left(d_{o i}\right)\right.} \\
\left.\left.+\mu_{2} \sum_{\substack{j=1 \\
i \neq j}}^{N} G_{o i}^{-1}(s) H_{i j}(s)\left(F_{i j}\left(y_{j}, t\right)\right)\right]\right\}
\end{gathered}
$$


where $e_{0}=y_{2}-y_{m}$. To rewrite the above cquation in a more concise form, wo define

$$
\begin{aligned}
\omega_{i} & =\left(r_{i}, w_{p i}^{T}, y_{i}, \omega_{f i}^{T}\right)^{T} \\
\eta_{i}^{i} & =\left(1-C_{p i}^{*}(s)\right)\left[G_{o i}^{-1}(s) \Delta_{a i}(s)+\Delta_{m i}(s)\right]\left(u_{i}\right) \\
\eta_{o}^{i} & =\left(1-C_{p i}^{*}(s)\right) C_{i_{i}}^{-1}(s)\left(d_{o i}\right) \\
\eta_{f}^{i} & =\sum_{\substack{j=1 \\
i \neq j}}^{N} G_{o i}^{-1}(s) H_{i j}(s)\left(F_{i j}\left(y_{j}, t\right)\right)
\end{aligned}
$$

where $\omega_{p i}=a_{i}(s) \lambda_{i}^{-1}(s)\left(u_{i}\right), w_{f i}=n_{i}(s) \lambda_{i}^{-1}(s)\left(y_{i}\right) \cdot a_{i}(s)=$ $\left[1, s, \cdots, s^{n_{i}-2}\right]^{7}$, and $\lambda_{i}(s)$ being an arbitrary monic polynomial of degree $n_{i}-1$ satisfying that $\lambda_{i}\left(s-q_{i}\right)$ is Hurwitz, to obtain

$$
\epsilon_{n i}=\frac{1}{c_{o}^{*}} M_{i}(s)\left(u_{i}-\theta_{i}^{* T} \omega_{i}+\mu_{1} \eta_{i}^{i}+\mu_{2} \eta_{f}^{2}+\eta_{o}^{i}\right)
$$

where $\theta_{i}^{*}=\left[i_{o i}^{*}, C_{p i}^{*}{ }^{T}, d_{b i}^{*}, D_{f i}^{*}{ }^{T}\right]^{T}$.

We carefully investigate the signals defined in (3.6) while keeping the assumptions ( 53$),(54)$, and the inequalities (3.2) in mind. Then, it is easy to find that if we define a normalizing signal for each subsystem as the state of the following differenlial equation:

$$
\dot{m}_{i}(t)=-\delta_{o i} m_{i}(t)+\delta_{1 i}\left(\left|u_{i}\right|+\sum_{\substack{j=1 \\ i \neq j}}^{N}\left|y_{j}\right|+1\right), \quad m_{i}(0)>\frac{\delta_{1 i}}{\delta_{0 i}}
$$

with $\delta_{0 i}, \delta_{1 i}>0$ and $\delta_{0 t}+\delta_{2 i}<\eta_{i}$ for some $\delta_{2 i}>0$, then the ratio between $\left|\eta_{i}^{i}\right|\left(\mathrm{or}\left|\eta_{f}^{i}\right|\right)$ and $m_{i}(t)$ must be bounded above [15]. which suffices for our subsequent construction of the sequential design algorithm.

\section{Practical Redesign Using Averaging Filter}

We first define the augmented signals $y_{1}$, and the atuiliary errors $\epsilon_{j_{i}}, j=1, \cdots, \nu_{i}$, as :

$$
\begin{aligned}
\epsilon_{1 i} & =\epsilon_{o i}+y_{1 i} i \quad y_{1 i}=M_{i}(s) L_{1 i}(s)\left(u_{1 i}-\gamma_{1 i}\right) \\
\epsilon_{2 i} & =\frac{1}{\ell_{1 i}(s)}\left(u_{2 i}\right)-\frac{1}{F(\tau s)}\left(u_{1 i}\right) \\
& \vdots \\
\epsilon_{\nu_{i} i} & =\frac{1}{\ell_{\nu_{i}-1, i}(s)}\left(u_{i}\right)-\frac{1}{F(\tau s)}\left(u_{\nu_{i}-1, i}\right)
\end{aligned}
$$

where $F(\tau s)$ can be any Hurwitz polynomial in $T s, \tau>0$, with degree at least two and $F(0)=1$. For clarity, here the averaging filter $\frac{1}{F(r s)}$ is chosen as $\frac{1}{(\tau s+1)^{2}}$ so that the bandwidth of the filter can be increased arbitrarily as the time constant $r$ approaches o. Now, the stop-by-step proof of the convergense of the revursive algorithm is initialized by investigating the dynamie: nodel of $1 i$ as :

$$
\epsilon_{1 i}=M_{i}(s) L_{1 i}(s)\left(u_{1 i}-\theta_{i}^{* T} \xi_{1 i}+\mu_{1} \eta_{1 i}^{i}+\mu_{2} \eta_{1 f}^{i}\right.
$$

$$
\left.+n_{i a}^{i}-\left(1-\frac{1}{c_{o i}^{*}}\right) \times 1 i\right)
$$

where $\xi_{1 i}=\frac{1}{c_{1}^{*} L_{1 i}(s)}\left(\omega_{i}\right), \eta_{1 i}^{i}=\frac{1}{c_{m_{1 i}}^{*} L_{1 i}(s)}\left(\eta_{i}^{i}\right), \quad \eta_{1 f}^{i}=$ $\frac{1}{c_{0,}^{*} i_{1 i}(s)}\left(\eta_{f}^{i}\right), \eta_{10}^{i}=\frac{1}{i_{a}^{*} L_{1,}(s)}\left(\eta_{o}^{i}\right)$, and $x_{1 i}=\frac{1}{L_{1 i}(s)}\left(u_{i}\right)$. In order for araalysis. we write down its associated state space representation as :

$$
\begin{aligned}
\dot{e}_{1 i}^{\prime}= & A_{1 i c_{1 i}^{\prime}}^{\prime}+b_{1 i}^{\prime}\left(u_{1 i}-\theta_{i}^{*} T_{1 i}-\psi_{i}^{*} \chi_{1 i}\right. \\
& \left.+\mu_{1, \eta_{1 i}^{i}}+\mu_{2} \eta_{1 f}^{i}+\eta_{10}^{i}\right) \\
e_{1 i}(t)= & d_{1 i}^{\prime} T_{1 i}^{\prime}(l),
\end{aligned}
$$

where the state vector $\epsilon_{1 i}^{\prime} \in R^{n_{i}}$ and the parameter $\psi_{i}^{*}=(1-$ $\left.\frac{1}{c^{*}}\right)$. Incorporating the definition of the normalizing signal in (4.2.8) and the SPR property of $M_{i}(s) L_{1 i}(s)$, we can design the auxiliary compensat ion signal $u_{1 i}=-\operatorname{sgn}\left(\epsilon_{1 i}\right)\left[M_{1 i} m_{i}(t)+M_{2 i}\right]$ with $M_{11}$ and $M_{12 i}$ satisfying inequalities :

$$
\begin{aligned}
\left|-\theta_{i}^{*} \xi_{1 i}-\psi_{i}^{*} x_{1 i}+\mu_{1} \eta_{1 i}^{i}+\mu_{2} \eta_{1 f}^{i}\right| & <M_{11 i} m_{i}(t)+\epsilon_{1 i}^{\prime}(t) \\
\left|\eta_{10}^{i}\right| & <M_{12 i}
\end{aligned}
$$

To verify the properness of choice (4.3.4), a simple Lyapunov function candidate $V_{1 i}=e_{1 i}^{\prime T} P_{1 i} e_{1 i}^{\prime}$ is picked, whose time derivative is given as follows :

$$
\begin{aligned}
\dot{V}_{1 i} \mid(4.4)= & -e_{1 i}^{\prime} T Q_{1 i} \epsilon_{1 i}^{\prime}+2 e_{1 i}\left(u_{1 i}-\theta_{i}^{*} T_{\xi_{1 i}}-\psi_{i}^{*} X_{1 i}\right. \\
& \left.+\mu_{1} \eta_{1 i}^{i}+\mu_{2} \eta_{1 f}^{i}+\eta_{10}\right) \\
\leq & -\epsilon_{1 i}^{\prime} T_{Q_{1 i} \epsilon_{1 i}^{\prime}}
\end{aligned}
$$

where $P_{1 i}, Q_{1 i}>0$ satisfy $P_{1 i} A_{1 i}+A_{1 i}^{T} P_{1 i}=-Q_{1 i}$ and $P_{1 i} b_{1 i}=c_{1 i}$ resulting from the SPR property of $M_{i}(s) L_{1 i}(s)$. The sirnilar arguments in [13] ensure that $e_{1 i}^{\prime}(t)$ converges to zero exponentially and the auxiliary error $e_{1 i}(t)$ drops to zero in finite time.

Completing the design of the first layex, we look into the second layer by rewriting the dynamic model for $e_{2 i}$ as :

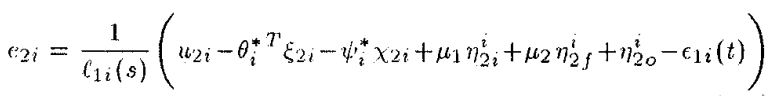

where $\xi_{2 i}, \chi_{2 \imath}, \eta_{2 i}^{i}, \eta_{2 f}^{i}, \eta_{20}^{i}$ are signals obtained by passing $\xi_{1 i}, \lambda_{1 i}, \eta_{1 i}^{i}, \eta_{1 f}^{i}, \eta_{10}^{i}$, respectively, through the strictly proper filter $\frac{\epsilon_{1 i}(s)}{F(\tau s)}$ and $\epsilon_{1 i}(t)=\frac{1}{F(\tau s) L_{2 i}(s)} M_{i}^{-1}(s)\left(e_{1 i}\right)$. For a general presentation, we derive in succession that

$$
\begin{gathered}
\epsilon_{j_{i} i}=\frac{1}{\ell_{j_{i}-1, i}(s)}\left(u_{j_{i} i}-\theta_{i}^{* T} \xi_{j_{i} i}-\psi_{i}^{*} \lambda_{j_{i} i}+\mu_{1} \eta_{j_{i} i}^{i}+\mu_{2} \eta_{j_{i} f}^{i}\right. \\
\left.+\eta_{j_{i} \sigma}^{2}-\epsilon_{j_{i}-1, i}(t)\right), \quad j_{i}=3, \cdots, \nu_{i},
\end{gathered}
$$

where $\xi_{y_{1} i}, x_{j, i}, n_{j_{1}, i}^{i}, n_{j+f}^{2}, n_{j, 0}^{2}$ are again sigmals obtained by filtering $\xi_{j,}-1, i, x_{j,-1, i}, \eta_{j_{1}-1, i}^{i}, \eta_{j_{i}-1, f}^{i}, \eta_{j_{1}-1,0}^{i}$ through the stable filter $\frac{\ell_{j_{1}-1, t}}{F(\tau s)}$,

$$
c_{j_{i}-1, i}(t)=\frac{1}{F(\tau s)} \ell_{j_{i}-1}(s)\left(\epsilon_{j_{i}-2}(t)\right)
$$




$$
+\frac{1}{F(\tau s)} \ell_{j_{i}-1}(s) c_{j_{i}-2}(s)\left(\epsilon_{j_{i}-2}(t)\right)
$$

and $u_{\nu_{i} i}=u_{i}$.

With regards to (4.6) and (4.7), the auxiliary compensation signals $u_{j_{i} i}, j_{i}=2, \cdots, \nu_{i}-1$, are chosen as :

$$
u_{j_{i} i}=-\operatorname{sgn}\left(e_{j_{i} i}\right)\left[M_{j_{i} 1 i} m_{i}(t)+M_{j_{i} 2 i}\right]
$$

where $M_{j, 1 i}$ and $M_{j i 2 i}$ satisfy

$$
\begin{gathered}
\left|-0_{i}^{* T} \xi_{j_{i} i}-\psi_{i}^{*} x_{j_{i} i}+\mu_{3} \eta_{j_{i} i}^{i}+\mu_{2} \eta_{j_{i} f}^{i}\right| \\
<M_{j_{i}{ }^{2} m_{i}(t)+\epsilon_{j_{i} i}(t)} \\
\left|\eta_{j_{i}}^{i}\right|<M_{j_{i} 2 i}
\end{gathered}
$$

Then, the finite-time convergence of signals $e_{j_{i}}(t), j_{i}=$ $2, \cdots, \nu_{i}-1$, are verified by incorporating the SPR properties of $\frac{1}{\ell_{j_{i}(i)}(s)}, j_{i}=2, \cdots, \nu_{i}-1$, and the fact that $\epsilon_{j_{i} i}(t), j_{i}=$ $1, \cdots, \nu_{i}-1$, are exponentially decaying signals. The last layer allows us to properly define the actual control force $u_{i}$ by observing that

$$
\begin{gathered}
\dot{\epsilon}_{\nu_{i} i}=-a_{\nu_{i}-1, i} \epsilon_{\nu_{i} i}+\left(u_{i}-\theta_{i}^{* T} \xi_{\nu_{i} i}-\psi_{i}^{*} x_{\nu_{i} i}+\mu_{1} \eta_{\nu_{i} i}^{i}\right. \\
\left.+\mu_{2} \eta_{\nu_{i} f}^{i}+\eta_{\nu_{i} 0}^{i}-\epsilon_{\nu_{i}-1, i}(t)\right)
\end{gathered}
$$

A proper choice of the control law will be

$$
u_{i}=\theta_{i}^{T} \xi_{\nu_{i} i}+\psi_{i} \chi_{\nu_{i} i}+S_{p i}
$$

with control parameters $\theta_{i}$ and $\psi_{i}$ (total with $\left(2 n_{i}+1\right)$ parameters) updated according to the following tuning formulae :

$$
\begin{aligned}
& \dot{\phi}_{i}=\dot{\theta}_{i}=-\Gamma_{i}\left(e_{\nu_{i} i} \xi_{\nu_{i} i}+\sigma \theta_{i}\right) \\
& \dot{\vartheta}_{i}=\dot{\psi}_{i}=-\beta_{i}\left(e_{\nu_{i}} \chi_{\nu_{i} i}+\sigma \psi_{i}\right),
\end{aligned}
$$

where $\vartheta_{i}=\psi_{i}-\psi_{i}^{*}, \Gamma_{i}=\Gamma_{i}^{T}>0, \beta_{i}>0$, and the switching control force $S_{p i}$ is chosen as :

$$
S_{p i}=-\operatorname{sgn}\left(e_{\nu_{i} i}\right)\left(M_{1 i} m_{i}(t)+M_{2 i}\right)
$$

with $M_{1 i} m_{i}(t)+\epsilon_{i}(t)>\left|\mu_{1} \eta_{\nu_{i} i}^{i}+\mu_{2} \eta_{\nu_{i} f}^{i}\right|$ and $M_{2 i}>\left|\eta_{\nu_{i},}^{i}\right|$.

The finite-time convergence of the signal $\epsilon_{\nu_{i} i}$ will be shown in [15] after we sketch the major results of this section in the following theorem for clarity.

Theorem 4.1:

Consider the system defined in section 3 with the control algorithm defined in this section. Under the assumptions (S1) $(\mathrm{S} 6)$, there exist $\tau^{*}>0$ such that for all $\tau \in\left(0, \tau^{*}\right)$, the following properties will hold for all $i \in \mathbf{N}$.

(a) $e_{j_{i}}$ approaches zero in finite time, $j_{i}=1, \cdots, \nu_{i}$.

(b) All signals in the closed-loop system are uniformly bounded.

(c) The output error $\epsilon_{o i}$ will converge exponentially to an arbitrarily small residual set whose size is a class $\mathrm{K}$ function of $\tau$ only.

(d) The magnitude of the switching gains $M_{1}, M_{2 i}$ can be chosen to be proportional to the order of the unmodeled dynamics and the unmodeled interconnections, namely, $\mu_{1}$ and $\mu_{2}$, and the order of the output disturbance, respectively.
Proof : Please refer to [1.5].

Remarks 4.1 : Since all the control gains can theoretically be chosen sufficiently large, the order of the unmodeled dynamics and the unmodeled interconnections, namely, $\mu_{1}$ and $\mu_{2}$, can be arbitrarily large without destroying the system stability. The tracking ejror vanishes as the design parameter $\tau$ becomes smaller (but $\tau$ can not be identically zero to prevent the use of the differentiators in the controller design) in spite of the degrees of the unmodeled dynamics, interconnections, and output disturbances.

\section{Simulation Example}

Consider the following interconnected dynamic systems.

$$
\begin{aligned}
& y_{1}=G_{o 1}(s)\left(1+\mu_{1} \Delta_{m 1}(s)\right) u_{1}+\mu_{2} H_{12}(s) y_{2} \\
& y_{2}=G_{o 2}(s)\left(1+\mu_{1} \Delta_{m 2}(s)\right) u_{2}+\mu_{2} H_{21}(s) y_{1}
\end{aligned}
$$

where the transfer functions are given as

$$
\begin{gathered}
G_{01}(s)=\frac{1}{s^{2}+s+1}, \Delta_{m 1}(s)=\frac{1}{s+10}, H_{12}=\frac{1}{\left(s^{2}+s+1\right)(s+2)} \\
G_{o 2}(s)=\frac{1}{s-1}, \Delta_{m 2}(s)=\frac{1}{s+5}, H_{21}(s)=\frac{1}{(s-1)(s+3)}
\end{gathered}
$$

$\mu_{1}=0.01, \mu_{2}=10$. The reference models chosen here are

$$
M_{1}(s)=\frac{24}{(s+4)(s+6)}, M_{2}(s)=\frac{5}{s+5}
$$

To implement the decentralized scheme in section 4 , we choose $L_{1 i}(s)=s+5$ such that $M_{1}(s) L_{11}(s)$ is SPR and the design time constant $\tau=0.01$. The simulation results are depicted in Figure 5.1. with reference inputs $r_{1}=r_{2}=1$.

\section{Conclusion}

In this paper, new decentralized MRAC algorithms for a class of interconnected uncertain dynamical systems have been presented. From the analysis provided, the main features of the developed approaches are :

(a) the algorithms are not completely decentralized, but the degree of computational complexity is about the same as those of the completely decentralized algorithms.

(b) the global stability of the adaptive systems is ensured in the presences of significant unmodeled dynamics, bounded output disturbances, and arbitrarily large interconnec tions, and the tracking errors drop to very small values as time goes on,

(c) each nominal subplant can be of any possible known relative degree,

(d) algorithm after practical redesign makes the proposed approach physically realizable and forces the output errors to diminish to zero by simply decreasing the time constant $\tau$,

(e) the improved transient performance is expected by incorporating the VSD.

The future research will be the reliability analysis of the proposed decentralized adaptive control algorithms under channel failures and the decentralized adaptive control of time-varying and/or nonlinear plants. 


\section{References}

[1] Annaswamy, A.M., and K.S. Narendra, "Stable Adaptive Systems", Prentice-Hall, 1989.

[2] Bodson, M. and S.S. Sastry, Adaptive Control : Stability, Convergence, and Robustness, Prentice-Hall, 1989.

[3] Datta, A. "Performance Improvement in Decentralized Adaptive Control : A Modified Model Reference Scheme", Proc. of 31th CDC, pp. 1346-1351, 1992.

[4] Fillippov, A.F., "Differential Equations with Discontinuous Right-Hand Side", Amer. Math. Soc. Transl., Vol. 42, pp. 199-231, 1964

[5] Gavel, D. T. and D. D. Šljak, "Decentralized Adaptive Control : Structural Conditions for Stability", IEEE Trans. Autom. Contr., Vol. AC-34, No. 4, pp. 413-426, 1989

[6] Hsu, L., "Variable Structure Model-Reference Adaptive Control (VS-MRAC) Using Only Input and Output Measurements : The General Case", IEEE Trans. Autom. Contr., Vol. AC-35, No. 11, pp. 1238-1243, 1990.

[7] Ioannou, P. A, "Decentralized Adaptive Control of Interconnected Systems", IEEE Trans. Autom. Contr., Vol., $A C-31$, No. 4, pp. 291-298, 1986.

[8] Otega, R. and A. Herrera, "A Solution to Decentralized Adaptive Stabilization Problem", Syst. \& Contr. Letter, pp. 299-306, 1993

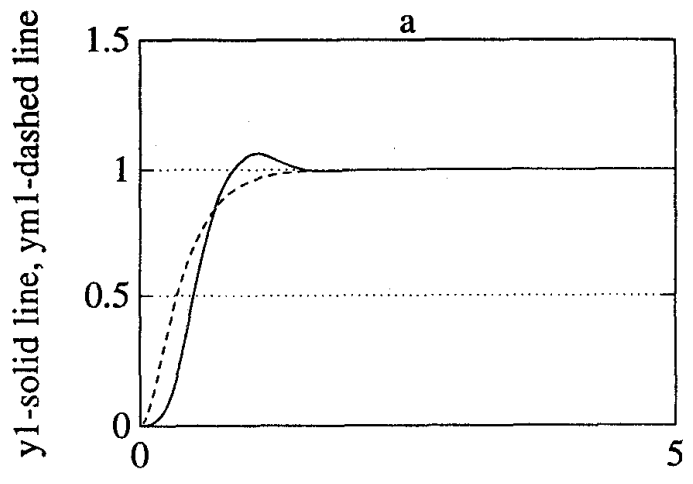

Time-sec

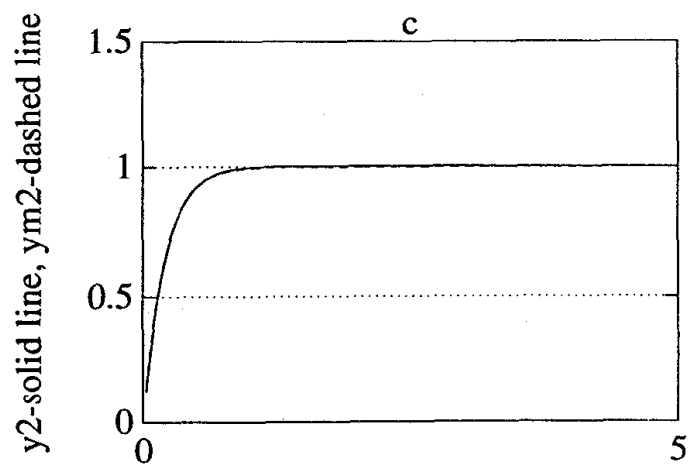

Time-sec
[9] Wu, A.C., L.C.Fu, and C.F.Hsu, "A New Decentralized Model Reference Adaptive Control for a Class of Interconnected Dynamic Systems Using Variable Structure Design", International Journal of Adaptive Control and Signal Processing, Vol. 7, pp. 33-43, 1993.

[10] Wu, A.C., L.C.Fu, and C.F.Hsu, "A New Robust Decentralized MRAC with Finite Time Perfect Tracking Properties for a Class of Interconnected MIMO Plants", Journal of Control Systems and Technology, Vol. 1, No. 1, pp. 33-42, 1993.

[11] Wu, A.C., L.C.Fu, and C.F.Hsu, "A New Decentralized Model Reference Adaptive Control for a Class of Interconnected Multivariable Plants", Proc. of ACC, pp. 1734-1735, 1991.

[12] Wu, A.C., L.C.Fu, and C.F.Hsu, "Robust MRAC for Plants with Arbitrary Relative Degree Using a Variable Structure Design", Proc. of ACC, pp. 2735-2739, 1992.

[13] Wu, A.C., L.C.Fu, and C.F.Hsu, "Practical Redesign of a Robust Model Reference Adaptive Controller for Uncertain Dynamic Systems", IEEE Trans. Autom. Contr., Revised, 1993.

[14] Wu, A.C L.C.Fu, and C.F.Hsu, "Variable Structure Design of MRAC for General Uncertain Plants". Journal of Control-Theory and Advanced Technology, Accepted, 1993.

[15] Wu, A.C. and L.C.Fu, "Decentralized MRAC for Largescale Uncertain Dynamic Systems Using Recursive Averaging Filter Design", Report, EE, NTU, 1993.

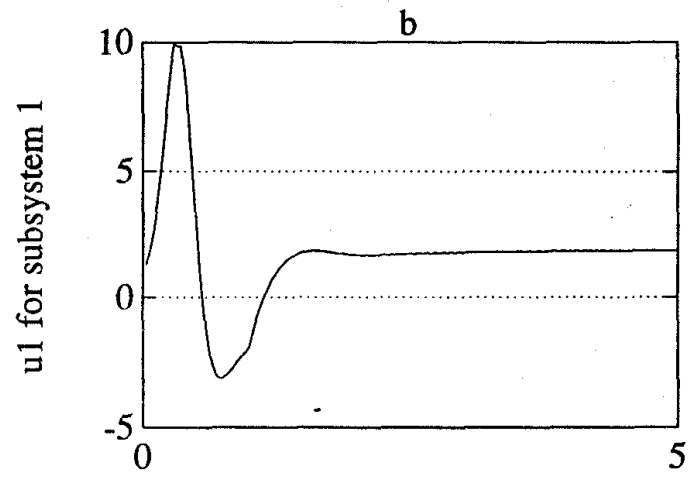

Time-sec

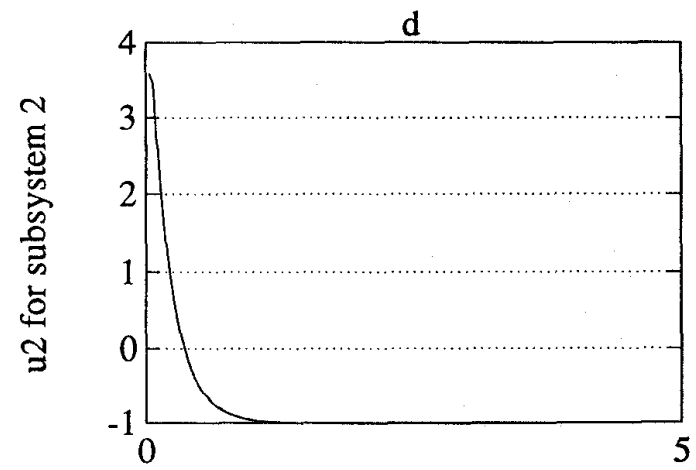

Time-sec

Fig. 5.1 Simulation plots for strongly connected $2 \times 2$ systems 